\title{
Towards a new face for Planetary Maps: Design and web- based Implementation of Planetary Basemaps
}

\author{
Andrea Nass $^{\mathrm{a},}$ *, Nicolas Manaud ${ }^{\mathrm{b}}$, Stephan van Gasselt ${ }^{\mathrm{c}}$, Trent M. Hare ${ }^{\mathrm{d}}$ \\ ${ }^{a}$ German Aerospace Center, Institute of Planetary Research, Berlin, Germany, andrea.nass@dlr.de \\ ${ }^{b}$ SpaceFrog Design, Toulouse, France,nicolas@spacefrog.design \\ ${ }^{c}$ National Chengchi University, Taipei,Taiwan,svg@nccu.edu.tw \\ ${ }^{d}$ US Geological Survey, Astrogeology Science Center, Flagstaff, US, thare@usgs.gov \\ * Corresponding author
}

\begin{abstract}
Modern planetary maps are designed either as stand-alone products or as part of a map series and are commonly distributed in the Portable Document Format (pdf). With the advent of web-based map technology and its standardization, experimental steps towards digital-only and dynamic map display for planetary cartography have been made. Commonly, maps design and concepts were limited to the arrangement (stacking) of various data layers while any deeper cartographic concepts or questions about usability seemed to be of secondary importance. We here present a new cartographic approach of creating a web basemap for Mars by refining and integrating commonly available data and by building on a cartographic concept. The underlying concepts we elaborated upon are, however, valid for any other planetary body and are applicable in the same or at least very similar way to any other solar system body. With this approach we want to demonstrate that for the creation of web maps in the planetary sciences the cartographic process does not stop at the collection and literal stacking of data, but that dynamic planetary maps can have a considerably higher communication value through concepts and design. As much as web maps are dynamic, so are concepts and new developments, and we tried to implement a web basemap reflecting the common understanding and picture that laymen as well as professionals might have about Mars.
\end{abstract}

Keywords: planetary cartography, web-mapping, cartographic concepts

\section{Introduction}

Planetary exploration looks back on a long history. It started with the world's oldest known depiction of astronomical phenomena (LDA-LSA, 2018) at least 4000 years ago and did not advance significantly until the early 1600 s due to the natural limitation of observations performed by the unaided eye. A significant leap came with the invention of the telescope around 1610, and with continuous improvements and the arrival of photographic processes at the beginning of the $20^{\text {th }}$ century, the number, variety and accuracy of planetary data has been steadily increasing, driven by technological, engineering, and processing innovations developed for every planetary mission. Resulting data are being used mainly

1. to gain first views on so far unknown planetary bodies,

2. to understand the shape and characteristics of a planetary body,
3. to increase terrestrial developments by drawing parallels to analogue processes on the Earth, and

4. to plan future autonomous or manned missions to further develop a better understanding of space and to find and evaluate potential resources based on former information and knowledge.

Visualization and communication of such information are achieved by cartographic products and thus hundreds of planetary maps have been crafted over the last 50 years. After all this time, however, one might wonder what image of a planetary body does this exploration effort leave in the human mind and how is that image reflected in a map? If people draw a mental map of the Earth, its dominant character is probably represented by a dichotomy between land and water surfaces, thus focusing on coastlines and colors. But how do we recognize the Moon, and what makes the Martian surface memorable? And how can we generate a basemap for planetary bodies that transports an unique image of the 
surface characteristics in our mind, comparable to Earth maps?

The work and map we present here is a contribution to a larger project under the name of OpenPlanetaryMap, which is an initiative to build an Open Planetary Mapping and Social platform for planetary scientists, space enthusiasts, educators and storytellers (Manaud et al., 2018). The main goal of this project is to make it easy to create and share location-based knowledge and maps of Mars and other planets of our Solar System. In this context, well-designed maps are needed as the base layers to use and enrich the overall user experience of the future platform.

Within this contribution, our cartographic design and web-based implementation handle these requirements, allowing to

- visualize the Martian surface as accessible as possible, and

- to serve as base map which can be superimposed by other topics and/or thematic information.

We aim to produce the first planetary vector-based and rendered basemaps that will provide a richer and more meaningful experience for viewers and map makers.

\section{Background}

\subsection{Planetary Maps for Science and the Public}

Maps of planetary bodies have been created systematically as soon as first telescopes became available in the early $17^{\text {th }}$ century. Most of these early sketches highlighted variations of surface reflectances of the lunar surface and Mars as the inner planets could not be observed against the Sun. It was not before the mid $18^{\text {th }}$ century that planetary surface depictions were created and could be termed actual maps, as they covered a consistent thematic topic, a graticule related to a geodetic reference system, and hosted a gazetteer-based nomenclature. Maps created today fall into one of three categories:

1. general purpose reference maps at small to relative large scale spanning at least several degrees of latitude and longitude and which represent surface albedo, topography and relief or the geology and physiography, commonly published as map sheets (e.g., the 1973 Lunar Chart Series at 1:1,000,000 published by the Defense Mapping Agency) or maps published as part of the USGS Scientific Map Investigation Series (Tanaka et al. 2014);

2. topical maps at larger scale accompanying research contributions published in conventional journals (e.g., van Gasselt et al. 2007, Jaumann et al., 2010);
3. dynamic digital maps published on online platforms using web and geospatial data services, e.g., USGS Mars Global GIS Mapping Application, (USGS, 2018a).

In the planetary sciences, maps are often freely accessible at no costs due to the concept of the underlying publicly funded exploration framework. From time to time, efforts are made to digitize some of the older analog maps and to transfer them to the digital domain (Hargitai et al., 2006).

\subsection{Web mapping: Services, Components and} Software as a Service

Web mapping (also called Web GIS) is a type of distributed information system, comprising at least a server and a client, where the server is a Geographic Information System (GIS) backend and the client is a web browser, desktop or mobile application. The interoperability of geospatial data, both in raster and vector form, across systems is made possible by the adoption and use of open standards defined by the Open Geospatial Consortium (OGC) (OGC, 2018). Today, web mapping is used in a wide variety of domains including: geosciences and environmental studies, defense and intelligence, Smart Cities and urban management and planning, emergency response and disaster management, and many more.

Google was the first to introduce slippy maps in 2005, and popularized web mapping through their ever evolving Google Maps web application and set of application programming interfaces (APIs).Since then, several new open-source and commercial web mapping Software as a Service (SaaS) have emerged (e.g.: OpenStreetMap, Mapbox, CARTO, Maptiler) and allow non-GIS experts to easily collect, visualize, manipulate and share their data in formats that are as slickly browsable. SaaS is a software distribution model in which a third-party provider hosts applications and makes them available to customers over the Internet.

\subsection{Planetary Interactive Mapping}

The Planetary Interactive GIS-on-the-Web Analyzable Database, PIGWAD, created by the USGS Astrogeology Science Center (ASC), was the first web-based GIS and initiative to support the planetary research community (Hare \& Tanaka, 2000). It has extended its services and capabilities overtime and evolved into what is now the Mercator GIS Lab, which provides web-based resources, including: a large collection of downloadable geospatial and map products; as well as OGC web services, and a Mars Global GIS mapping application.

The USGS's ASC has paved the way for the emergence of other planetary web-based mapping interfaces: initially map-based data search and access portals (e.g., PDS 
Geosciences ODE, REACT-QuickMap, iMars), but later developed and used for scientific research purpose (PlanetServer, MARSWEB), for landing site selection and science operations (JMARS), and more recently for outreach and education (NASAs Solar System Trek Suite, Google Maps/Earth, JMARS). In collaboration with NASA researchers at Arizona State University and USGS's ASC, Google contributed to make planetary maps popular on the web with their Google Mars/Moon interfaces in 2011.

However, since then, even today's state-of-art planetary web mapping applications still use traditional global mosaicked raster data as basemap. Zooming in and out such basemaps allows a viewer to see planetary surfaces at different scales and levels of detail; but it does not provide additional contextual information (derived or interpreted), and makes it difficult to overlay other datasets and create readable visualizations.

\section{Cartographic Concept and Data Model}

\subsection{General Visualization Concept}

In order to arrive at a valid, self-consistent and usable map layout which is re-usable as basemap, we have developed a cartographic concept built on a number of key questions:

1. what kind of information do we want to communicate, i.e. which picture do we want to draw of Mars?

2. who are the recipient and target audience?

3. how can we maximize functionality and aesthetics with as few datasets as possible?

The latter question usually highly depends on the tools that are available within a given mapping platform, however, the conceptual design is supposed to be valid, complete and independent of the actual implementation platform.

In section 4 we will discuss how these concepts were physically implemented within an actual cartographic data model. Despite being used for several years now, one of the major challenges in modern web map design is that it still lacks targeted cartographic rules as well as representative experiences and best practices, and it would not be a healthy approach to transfer rulesets for analog cartographic design directly to the digital world. Most of contemporary implementations will need to stand the test of time to see how people will work with them, and how people will perceive digital map implementations. However, conventional rules and best practices are certainly a healthy starting point to think about the design process of web maps.
We here address the question of what is needed to represent a planetary surface by tools of cartographic visualization to make it accessible for a broad audience, i.e. the scientific as well as laymen community alike. Based on the primary aim the required contents and individual visualizations can be derived and further developed. The second key value for the cartographic concept deals with the datasets on which the map presentation will be based upon. Due to the lack of different global high-quality datasets to choose from, the selection was relatively straightforward. However, the conceptual design has to cope with new datasets that might come up in the future. One implementation constraint was given and that did not allow us to use any raster image data as the CARTO platform as our choice of implementation and testing requires vector data.

An easily accessible global picture of Mars might be represented by one or more of its three global characteristics:

1. the typical red Mars color (hue),

2. the characteristic distribution of variations of lightness values, also known as surface albedo features, i.e., the (physical) reflectance of surface materials, and

3. the global topography with its characteristic dichotomy separating highlands from lowlands and rougher-surface areas from smoother ones.

All of these features are discernible and distinguishable on larger as well as smaller map scales alike.

The problem with the first two characteristics is that for the first one no calibrated color representation exists and thus, the typical Mars color varies from a sandy brown to a dark brown-red depending on the way color was derived from the data. The challenge with the second characteristic is that the distribution of albedo features is highly variable over the year and this reflects the extent of the polar caps as well as many of the low-albedo features in the plains. Consequently, there is no commonly valid concept of one characteristic picture of Mars. It might be for that reason, that common global maps of Mars usually lack albedo features. We consider this as a major shortcoming as several dozen features on the Martian surface were in fact historically named because of their dominant and clearly visible albedo. Therefore, color and its variations provide an intuitive picture of Mars which can already be verified at low resolution and small map scales. Beside this, further individual large topographic and tectonic features such as the volcanic bulges of Tharsis in the Eastern hemisphere with the Solar Systems highest volcano, large valleys, such as the Valles Marineris, as well as plains and huge impact basins characterize the global view of the surface 
and their implementation on a map might add to an easier perception.

In order to create and depict an accessible global view of Mars, we had a number of options for developing a concept to start with. Classically for Mars maps, a grayscale shaded-relief representation is superimposed by a layer representing a hypsometric tinting of the underlying terrain model data using a detailed rainbowspectrum color scale. As alternative, the subdued shaded relief representation is superimposed by, or combined with a global (ortho-) image map, commonly provided by data from early 1980s or 1990s instruments. Both views provide a valid characteristic picture that we have of Mars and both have their individual limitations. The image-mosaic-relief representation is limited with respect to offering a quantitative approach to extract elevation values, and the color-relief version lacks any natural color or albedo feature and can best be described as representation of physical measurements, which is additionally supported by the rainbow spectrum that is easily mentally connected to laboratory measurements.

When thinking of visualization of the main characteristics we decided to take a look at larger-scale topographic map series from the Earth with scales of 1:10,000 to 1:100,000. Here, natural areal features are separated into roughly three main areas: land with (green) and without (white) vegetation, and water surfaces, which by their extent define coastlines and continental shapes. This minimalistic classification is unobtrusive and a perfect fit for topographic maps as it allows placement of contour lines and additional symbols. The color spectrum (hue) of the Martian surface is red to brown depending on the actual model that has been used to extract "true" colors of Mars. The brightness values range from extremely low values close to $5 \%$ to high values close to $90 \%$ at the polar caps. However, despite the large range of brightness values, it is possible to classify the surface into about five different zones which form large connected area in the northern highlands and more dispersed areas in the southern highlands.

Our concept had to face the challenge to not only depict Mars in an accessible and accurate way using limited data but also we had to make sure we are able to replace the representation of raster data by vector representations, i.e., finding a discretization of continuous pixel-based data without reproducing each pixel value using vector geometries.

In our concept we wanted to make use of typical surfacecolor values to define a hypsometric color range to be used for coding classified topographic values. As we find it important to also implement typical albedo variations without obscuring hypsometric tinting we decided to create an additional non-obscuring layer indicating zones of different surface albedos. We decided against the classical rainbow spectrum tinting for the Martian topography as this information -- although very popular -is not natural, and it owns a significant dominance which does not allow to add any other type of information. Also, the rainbow-spectrum with blue as lowest values might imply the existence of water in the lowlands of the Northern Hemisphere of Mars.

Contour lines, when well balanced, allow to represent quantitative topographic information in a more subtle way by allowing more space for other information. The density of contour lines along topographic dominant, i.e., high-relief areas, will create a density contrast when compared to low-relief areas. The higher density of contour lines will make it easier to spot characteristic global features, such as the escarpment of the dichotomy boundary, the larger valley systems or the volcanic rises, which we consider a useful side effect helping to access the map of Mars.

At the time of writing and developing, a number of global data sources are available which allow us to extract information and comparable scales. As no noteworthy ground data coverage is available except for a few rover locations, this map representation is based on orbital data only. Datasets and applied methods will describe in the following sections and the actual implementation will be summarized in section 4 .

\subsection{Data Processing and Selection}

We used laser altimeter topography data based on Mars Global Surveyor Mars Orbiter Laser Altimeter (MGS$M O L A)$ due to its near-global coverage (Albee et al., 2001; Smith et al., 2001; Zuber et al, 1992) with 231 $\mathrm{m} / \mathrm{px}$ (256 px/deg) near the poles and $463 \mathrm{~m} / \mathrm{px}$ (128 $\mathrm{px} / \mathrm{deg}$ ) for mid- and equatorial latitudes. Global datasets are made available via the Planetary Data System (PDS).

Basic processing and conversion were carried out using the publicly available Integrated Software for Imagers and Spectrometers 3 (ISIS) maintained and provided by the US Geological Survey (USGS) (Becker et al., 2013; Keszthelyi et al., 2013). Further processing was performed within ESRIs ArcGIS platform and tools making use of OSGeo's Geospatial Data Abstraction Library (GDAL). This includes format filtering, conversions and derivation of additional data products (i.e., slope maps, contours).

Topography data were complemented by global 2channel image data obtained during a geodesy survey by the wide angle instrument of the MGS Mars Orbiter Camera (MGS-MOC) assembly onboard Mars Global Surveyor (Caplinger \& Malin, 2001). 
Despite its age and severe noise artefacts in the southern hemisphere due to a dust storm event, this dataset provides thus far the most complete and most homogeneous coverage of the planet in two color channels, red and blue. We are anticipating, however, that the results from the Mars Express High Resolution Stereo Camera (HRSC) instrument will soon provide global data at higher spatial and spectral resolution for both, topography and imaging (Neukum et al., 2004; Jaumann et al., 2006).

For the albedo representation we decided to incorporate spectral information from the Mars Thermal Emission Spectrometer (TES) Bolometric Albedo Global Map provided by the USGS with a resolution of $7410 \mathrm{~m} / \mathrm{px}$ (Christensen et al., 2001).

Due to several image artefacts additional low-pass filtering has been applied which essentially reduced the resolution to below $25 \mathrm{~km} / \mathrm{px}$. Despite these artefacts we preferred to use the global albedo map as we consider this a product with physically meaningful values. Reflectance values from optical imaging devices are currently not corrected for atmospheric effects and are considered less useful for our purpose.

The global nomenclature used here is based upon data approved and provided by the International Astronomical Union (IAU) gazetteer which was later adjusted and refined by the USGS (cf. Hunter et al., 2016). The IAU responds to proposals from the community via a task group and the Working Group for Planetary System Nomenclature (WGPSN) in a bottom-up fashion (IAU, 2018). Data are provided as static PDF maps, text files as well as in the ESRI shapefile format. For Mars, the official catalog consists of close to 1900 entries across the planet with label entries, their origin, their center geographic coordinates and their bounding boxes including links to meta information. Given the density of impact crater structures on planetary surfaces it comes as no surprise that $60 \%$ of all planetary nomenclature entries are labels for impact craters and consequently we adopted them as our priority for implementing labelling.

\subsection{Incorporation into Web Map Platforms}

New modern web-based mapping platform/SaaS, such as CARTO, Mapbox, or Maptiler, popularized map making on the web. Unlike commercial applications (e.g. Google Maps, ESRI, etc.), these platforms all are, and rely on, open source software and standards (OSGeo); and use open geospatial database (OpenStreetMap).

In the context of the OpenPlanetaryMap project, we have chosen CARTO as our underlying platform. We use it to manage, analyze and visualize our geospatial datasets; and to create maps using and styling these datasets, using the CartoCSS styling language/interface. A dedicated CARTO Map API can then be used to serve these maps as basemap raster-tiles (following standard XYZ tiling scheme) that can be easily used in web applications using mapping javascript library (Leaflet, OpenLayers, ...). All vector geospatial datasets (often in Shapefile format) are imported into CARTO. Although CARTO can handle different map projections by re-projecting datasets automatically and making them available in geodetic projection (EPSG:4326, WGS84); available basemaps and map creation are done in Web Mercator projection. Global raster datasets, such as hillshade, are re-projected into the Web Mercator projection (EPSG:3857) using GDAL, tiled following standard XYZ tiling scheme, and served from a AWS S3 server.

The CARTO Map API allows us to configure our basemap end-product so that it can be made available in different combination of layers (styled vector datasets or rasters). For example, the nomenclature layer can be accessed separately and be overlapped on top of any raster layer.

\section{Interpretation and Discussion}

We used MOLA data as basic topography dataset and to derive further products. Due to its popularity in the planetary sciences, we derived a hillshade dataset as qualitative basic relief representation with main illumination from the top-left (NW). As conventional hillshade derivatives lack a certain depth and suffer from an unbalanced representation of lit and unlit areas with too strong contrasts, we decided to develop a multilayer and multidirectional hillshade representation in combination with slope information which takes into account the severe data artefacts commonly seen on planetary surfaces. The resulting dataset was subsequently re-classified to reduce its complexity, exported as vector dataset and generalized in order to be usable at various dynamic map scales.

In addition, we exported a contours dataset based on a reduced resolution topography raster model to subdue any higher-level artefacts introduced by the measurement characteristics. Contour lines are extracted and processed to (a) reduce the number of vertices (generalization), and (b) smoothen their shape. This process is iterative and requires some investigations into the what we considered a visually acceptable representation. Also, the process needs to be performed automatically on a global scale without producing topological nonsense (e.g., intersections). The contour level was initially set to 100 $\mathrm{m}$ with index contours at $500 \mathrm{~m}$. Contour lines shorter 

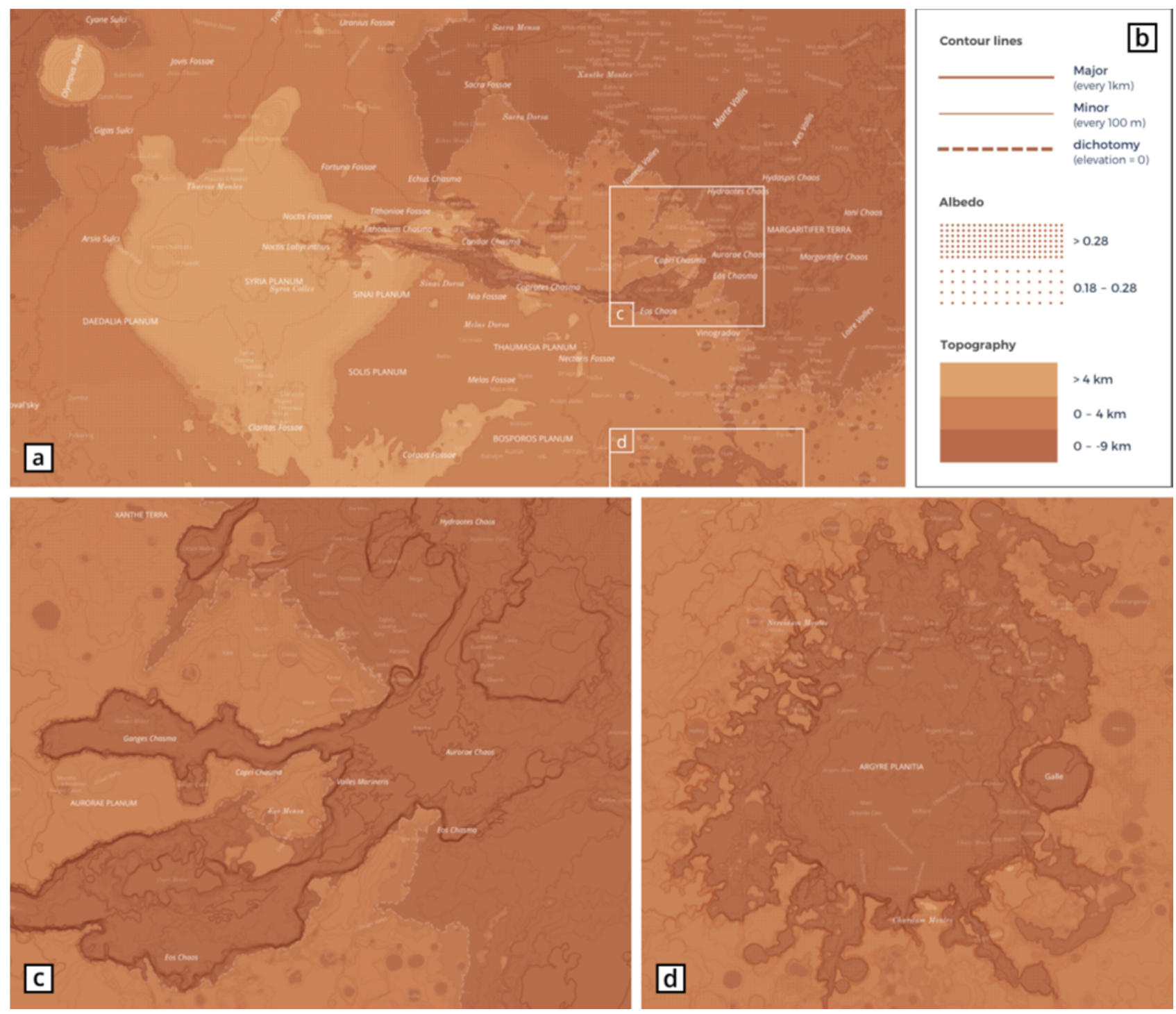

Figure 1 Sample screenshots from the CARTO web map of Mars. [a] the Eastern volcanic Tharsis rise with the Valles Marineris structure and Chryse Planitia outflow area (zoom level 4, scale is about 120,000,000); [b] sample map legend; [c] the Eastern Valles Marineris area Xanthe Terra; the area spans about 30 degrees of longitude translating to $1800 \mathrm{~km}$ at the equator (zoom level 6, scale is about 1:20,000,000); [d] the Argyre impact basin at high southern latitudes (zoom level 5, scale is about 1:25,500,000), Map projection is Web Mercator which causes non-linear relationships between zoom levels and map scales.

than $100 \mathrm{~km}$ were eliminated to reduce noise. At highrelief areas such as the Tharsis region, the Elysium volcanoes and parts of the Valles Marineris and Noctis Labyrinthus region contour lines were eliminated manually. For the first implementation we made use of a contour dataset processed and made available by the USGS. The current concept is static in a way that it builds on pre-defined contour datasets. These pre-defined contour datasets are, however, globally defined although it would be a much nicer approach to define contour spacing based on the local relief and map scale (zoom level) as suggested in the literature (e.g., Hake et al., 2001). This would provide optimum results for highrelief areas (fewer contours) and lowlands plains where the contour spacing can be reduced (cf. Figure 1a-d).
While the implementation of the topography concept is straightforward in terms of incorporation of data using the CARTO platform, its realization in terms of preprocessing is challenging due to an abundance of data and interpolation artefacts in the archived raster datasets. Unfortunately, the MOLA dataset cannot be processed from raw data levels with the same quality as it was manually corrected and visually improved by the MOLA science team without being documented. Readouts of the original track data and application of an improved interpolation is possible but the results will contain all erroneous tracks. Still, the manually improved raster dataset has flaws as seen by numerous jagged lines in NS direction and generally considerable jumps in the level of detail when comparing along track and across track space. By vectorizing and applying generalization and smoothing of contour lines it is easy to create topological 
errors that are hard to eliminate once they are introduced. The amount of required smoothing to eliminate these errors causes a deterioration of resolution and will still not provide satisfactory results. Subsequent hypsometric tinting has been carried out using 50x50 pixel average sampling window over the global MOC color mosaic. Low-lightness values were assigned to topographically low area, and brighter areas were assigned to higher elevations in order to create a similar effect as commonly applied for physiographic maps of the Earth. Previous attempts of applying hypsometric tinting using a non rainbow-spectrum have been made (USGS, 2018b) and also visually very distinct and accessible it found limited use only. The current implementation in CARTO shows the proof of concept and requires more differentiation of elevation classes without introducing too much blur due to the limited hue range.

For the albedo, i.e. variation of dark and bright surface areas, we made use of the TES albedo map provided by the USGS and applied a low-pass filter before performing a threshold classification using boundaries based on visual inspection. As the surface of Mars shows some variations of albedo as discussed above, it can easily be broken down to no more than five contiguous areas that could be considered as representative. Based on the average areal lightness we experimented with pattern fills of various line pattern densities. The challenge we encountered here is not related to the principle technical implementability but to the problem that dynamic zooms require a clever adaptation of fill pattern densities that are at this stage not automatically being done by CARTO. In order to accomplish a dynamic adaptation a set of various fill pattern representations would have to be created. This work has, however, not been completed yet. At small scales and given print resolution patterns are not well discernible (cf. Figure 1a, c).

For the nomenclature and its first implementation we decided to select and extract the following features from the nomenclature shapefile: impact craters $>200 \mathrm{~km}$ in diameter (craters), features with a commonly high lengthto-width ratio such as valleys (lat. vallis, valles) $>200 \mathrm{~km}$ (in extent) as well as tectonic features such as grabens (lat. fossa, fossae), volcanic structures (lat. tholus, tholi; mons, montes and patera. paterae) due to their areal dominance, and finally, features with a larger areal extent such as plains (lat. planitia, planitiae as well as planum, plana and terra, terrae features). This selection will transport as much details as needed, but it is not too overloaded for a global map. The revised dataset provided by USGS does not only provide the areal extent of each feature but also a rotation angle allowing to better place and distribute features on the map. At this moment, however, more advanced placement options, such as alignment to curved objects and paths are lacking which, however, will be hard to implement given the fact that dynamic zoom levels require updates along object paths whenever the user changes the zoom level. Also, there are several improvements to be made regarding label sizes and character spacing (cf. Figure 1a-b, d).

\section{Summary and Outlook}

Using CARTO as our geospatial datasets storage and visualization platform and CartoCSS as styling language, we have been able to implement key elements of our cartographic concept and to produce the first vector-based basemap for Mars.

At this stage we have not been able to implement all our conceptual elements on a mature level as the vectorization of global raster data poses a number of challenges on itself. This is mainly related to relatively higher levels of noise, in particular in topographic and spectral data. However, once an appropriate filtering has been applied and deterioration of the original data resolution can be accepted the conversion and implementation is possible as tested for a number of regionally confined cases.

Our aim to provide a vector-rendered basemap could accomplice in the first implementation by using CARTO. CARTO serves our basemap datasets both as raster and vector tiles, but currently its Map API does not allow for vector rendering. As CARTO is evolving towards vector rendering technology and Mapbox GL to render interactive maps, we will explore this new capability. We will also assess Mapbox and Maptiler as a tool to design our future vector-rendered basemaps.

\subsection{Acknowledgements}

This development project has made use of the USGS Integrated Software for Imagers and Spectrometers (ISIS). The authors wish to thank the CARTO team for providing free access to their platform and support for implementing and testing our map.

\section{References}

ACT (2018). ACT-REACT-QuickMap. 30 Nov. 2018, www.actgate.com/quickmap.html.

Albee, A.L., Arvidson, R.E., Palluconi, F., and Thorpe, T. (2001). Overview of the Mars Global Surveyor mission. Journal of Geophysical Research, 106(E10): 23, 29123, 316.

Becker, K.J., Anderson, J. A., Weller, L.A., and Becker, T.L. (2013). ISIS Support for NASA Mission Instrument Ground Data Processing Systems. $44^{\text {th }}$ Lunar Planetary Science Conference, Houston, TX, US, LPI Contribution 2083, \#2829. 
Caplinger, M. and Malin, M. (2001). The Mars Orbiter Camera Geodesy Campaign. Journal of Geophysical Research, 106(E10).

Christensen, P.R., Bandfield, J. L., Hamilton, V. E., Ruff, S. W., Kieffer, H. H., Titus, T. N., Malin, M. C., Morris, R. V., Lane, M. D., Clark, R. L., Jakosky, B. M. Mellon, M. T., Pearl, J. C., Conrath, B. J., Smith, M. D., Clancy, R. T., Kuzmin, R. O., Roush, T., Mehall, G. L., Gorelick, N., Bender, K., Murray, K., Dason, S., Greene, E., Silverman, S., and Greenfield M. (2001). Mars Global Surveyor Thermal Emission Spectrometer experiment: Investigation description and surface science results. Journal of Geophysical Research, 106, 23,823-23,872. doi:10.1029/2000JE001370.

van Gasselt, S., Hauber, E. and, Neukum G. (2007). Coldclimate modification of Martian landscapes: A case study of a spatulate debris landform in the Hellas Montes Region, Mars. Journal of Geophysical Research, 112(E9), doi:10.1029/2006JE002842.

Hake, G., Gruenreich, D., and Meng, L. (2001). Kartographie: Visualisierung raum-zeitlicher Informationen. 8. ed., 617 pp. (de Gruyter).

Hargitai, H., and Pitura, M. (2018). International Catalog of Planetary Maps. $49^{\text {th }}$ Lunar Planetary Science Conference, Houston, TX, US LPI Contribution 2083, \#2608.

Hargitai, H. (2006). Planetary Maps: Visualization and Nomenclature. Cartographica 41(2): 149-167, doi:10.3138/9862-21JU-4021-72M3.

Hare, T.M., and Tanaka, K. L. (2000). PIGWAD - New Functionality For Planetary GIS On The Web. $31^{\text {th }}$ Lunar Planetary Science Conference, Houston, TX, US, LPI Contribution 2083, \#1889.

Hunter, M.A., Fortezzo, C.M., Hayward, R.K., Hare, T.M. (2016): Feature-linked Annotation of Lunar Nomenclature. $47^{\text {th }}$ Lunar Planetary Science Conference, Houston, TX, US LPI Contribution 2083, \#1558.

IAU (2018). Gazetteer of Planetary Nomenclature. Planetary Names, 30 Nov 2018. planetarynames.wr.usgs.gov/.

LDA-LSA (2018). The Nebra Sky Disk. 08 Dec 2018, State Office for Heritage Management and Archaeology. www.ldalsa.de/en/nebraskydisc.

Jaumann, R., Nass, A., Tirsch, D., Reiss, D., and Neukum, G. (2010). The Western Libya Montes Valley System on Mars: Evidence for episodic and multi-genetic erosion events during the Martian history. Earth and Planetary Science Letters, 294 (3-4): 272-290, doi:10.1016/j.eps1.2009.09.026.

Jaumann, R., Neukum, G. Behnke, T., Duxbury, T.C., Eichentopf, K., Flohrer, J., van Gasselt, S., Giese, B., Gwinner, K., Hauber, E., Hoffmann, H., Hoffmeister, A., Koehler, E., Matz, K-D., McCord, T.B., Mertens, V., Oberst,, J., Pischel, R., Reiss, D., Ress, E., Roatsch, T., Saiger, P., Scholten, F., Schwarz, G., Stephan, K., Waehlisch, M., and the HRSC CoInvestigator Team (2006). The High-Resolution Stereo Camera (HRSC) experiment on Mars Express: Instrument aspects and experiment conduct from interplanetary cruise through the nominal mission. Planetary and Space Science, 55:928-952.
Keszthelyi, L., Becker, T., Sides, S., Barrett, J., Cook, D., Lambright, S., Lee, E., Milazzo, M., Oyama, K., Richie, J., Saleh, R., Shinaman, J., Sucharski, T., Titus, T., Walldren, J., and Weller, L. (2013). Support and Future Vision for the Integrated Software for Imagers and Spectrometers (ISIS). $44^{\text {th }}$ Lunar Planetary Science Conference, Houston, TX, US, LPI Contribution 2083, \#2546.

Manaud, N, Nass, A., Lewando, M., van Gasselt, S., Rossi, A.P., Hare, T., Carter, J., and Hargitai, H. (2018). OpenPlanetaryMap: Building the first Open Planetary Mapping and Social platform for researchers, educators, storytellers, and the general public. European Planetary Science Congress 2018, 12, EPSC2018-78.

Neukum, G. and Jaumann, R. (2004). HRSC: the High Resolution Stereo Camera of Mars Express. In: Mars Express: the Scientific Payload (Wilson, A. ed), ESA SP-1240, Noordwijk, Netherlands: 17-35.

OGC (2018). W3C and OGC Spatial Data on the Web Working Group publishs First Public Working Draft of its Best Practices. Press Releases, 30 Nov. 2018. www.opengeospatial.org/pressroom/pressreleases/2358.

Smith, D.E., Zuber, M.T., Frey, H.V., Garvin, J.B., Head, J.W., Muhleman, D.O., Pettengill, G.H., Phillips, R.J., Solomon, S.C., Zwally, H.J., Banerdt, W.B., Duxbury, T.C., Golombek, M.P., Lemoine, F.G., Neumann, G.A., Rowlands, D.D., Aharonson, O., Ford, P.G., Ivanov, A.B., Johnson, C.L., McGovern, P.J., Abshire, J.B., Afzal, R.S., and Sun, Xiaoli (2001). Mars Orbiter Laser Altimeter Experiment summary after the first year of global mapping of Mars. Journal of Geophysical Research, 106(E10):23,68923,722.

Tanaka, K.L., Skinner, J.A., Jr., Dohm, J.M., Irwin, R.P., III, Kolb, E.J., Fortezzo, C.M., Platz, T., Michael, G.G., and Hare, T.M., (2014). Geologic map of Mars: U.S. Geological Survey Scientific Investigations Map 3292, scale 1:20,000,000, 43 pp.

USGS (2018a). Mars Global GIS Mapping Application. 07 Dec 2018, webgis2.wr.usgs.gov/Mars_Global_GIS/.

USGS (2018b). Topographic Map of Mars. 10 Dec 2019. pubs.usgs.gov/imap/i2782/.

Vargas, C., Muller, J.-P., and Morley, J.G. (2010). MARSWEB: A GIS based web 2.0 mapping application to measure impact craters on the surface of Mars. European Planetary Science Congress 2010, 5, EPSC2010-881.

Walter, S.H.G., Muller, J.P., Sidiropoulos, P., Tao, Y., Gwinner, K., Putri, A.R.D., Kim, J.R., Steikert, R., van Gasselt, S., Michael, G.G., Watson, G., Schreiner, B.P. (2018). The WebBased Interactive Mars Analysis and Research System for HRSC and the iMars Project. Earth and Space Science, 5(7):308-323, doi:10.1029/2018EA000389.

Zuber, M. T., Smith, D. E., Solomon, S. C., Muhleman, D. O., Head, J. W., Garvin, J J. B,. Abshire, B., and Bufton, J.L. (1992). The Mars Observer Laser Altimeter investigation. Journal of Geophysical Research, 97, 7781-7797. 